\title{
Pharmaciens œuvrant à la sécurité des patients
}

par Patricia Macgregor

$\mathrm{D}$ ans le système de santé, l'objectif est d'offrir de meilleurs soins aux patients; des soins fondés sur des normes et axés sur les résultats. Cet objectif repose sur le double principe voulant que tous les patients aient droit à des soins sécuritaires ainsi qu’à la meilleure pharmacothérapie. Je suis convaincue que tous les pharmaciens croient en ce principe, tout comme je suis persuadée que nous nous efforçons de l'appliquer. Or, faisons-nous tout en notre pouvoir pour garantir la sécurité des patients dont nous avons la charge et pour nous assurer qu'ils reçoivent les soins les mieux adaptés à leurs besoins? Nous avons tous un rôle à jouer pour ce qui est d'assurer leur sécurité et d'éviter les préjudices, mais l'endossons-nous pleinement?

De nouveaux modèles de soins offrent aux pharmaciens l'occasion d'être au premier plan pour ce qui est de l'amélioration des services aux patients et des résultats cliniques. Les leaders en pharmacie doivent sensibiliser davantage l'opinion (à l'intérieur et à l'extérieur de la profession) à la valeur qu'offrent les pharmaciens comme fournisseurs de soins, non seulement à titre de distributeurs de médicaments. De leur côté, les pharmaciens doivent faire la preuve qu'ils ajoutent une valeur aux soins des patients.

Posons-nous la question : souhaitons-nous que notre profession soit reconnue pour l'impact des incidents liés aux médicaments et des défaillances du système... ou pour les améliorations que nous apportons à la sécurité et à l'état de santé de nos patients? Consacrons nos efforts à promouvoir les meilleurs soins aux patients.

Les pharmaciens ne sont pas seuls à œuvrer pour l'amélioration de la sécurité des patients et de la qualité des soins. Des organismes de soins de santé accordent énormément d'attention à ces aspects de performance et exigent qu'on en assume la responsabilité. D'ailleurs, les ordres professionnels et des réseaux et organismes voués à la sécurité (notamment l'Institut canadien pour la sécurité des patients (ICSP), l'Institut pour la sécurité des médicaments aux patients du Canada et le Children's Hospitals' Solutions for Patient Safety) en font tout autant. Les orientations de ces organismes et réseaux, ainsi que celles d'organismes semblables, concordent avec celles de notre profession et de la Société canadienne des pharmaciens d'hôpitaux (SCPH). Sous le domaine clé de la Pratique des pharmaciens, le plan stratégique de 2015-2018 de la SCPH comportera de nouvelles occasions de partenariat visant à améliorer l'application des connaissances et les résultats thérapeutiques. Parmi les partenariats envisagés, la $\mathrm{SCPH}$ s'associera à d'autres parties prenantes du Plan d'action intégré en sécurité des patients de l'ICSP afin de mettre en œuvre des actions en lien avec la sécurité des soins chirurgicaux, la sécurité des médicaments ainsi que la prévention et le contrôle des infections.

Enfin, avec l'avènement des technologies pour la distribution des médicaments, les rôles traditionnels des pharmaciens et des techniciens sont remplacés par des perspectives grandissantes dans les milieux cliniques en collaboration, notamment un potentiel croissant pour la pratique avancée et la spécialisation. La gestion responsable, en ce qui a trait à la valorisation et aux décisions thérapeutiques, sera de plus en plus importante. D'ailleurs, les pharmaciens sont particulièrement bien placés pour s'attaquer aux défis propres au transfert des soins entre les hôpitaux ou les systèmes de soins de santé et le milieu communautaire. Ils ont aussi la chance de façonner leurs rôles dans l'optimisation et l'observance pharmacothérapeutiques, la prévention des maladies et des incidents liés à la sécurité, l'adoption des meilleures pratiques, l'autogestion des soins et la surveillance des patients ainsi que dans les soins de santé en collaboration. Ensemble et avec les autres membres de l'équipe de soins de santé, nous pouvons faire avancer les choses.

[Traduction par l'éditeur]

Patricia Macgregor, B. Sc., R. Ph., M. R. Pharm. S., M. H. Sc., C.H.E., est présidente sortante et agente de liaison pour la vision de la Société canadienne des pharmaciens d'hôpitaux. 